\begin{tabular}{|c|c|c|c|c|c|c|}
\hline \multirow{4}{*}{ Impact Factor: } & ISRA (India) & $=3.117$ & SIS (USA) & $=0.912$ & ICV (Poland) & $=6.630$ \\
\hline & ISI (Dubai, UAE & $=0.829$ & РИНЦ (Russia & $=\mathbf{0 . 1 5 6}$ & PIF (India) & $=1.940$ \\
\hline & GIF (Australia) & $=0.564$ & ESJI (KZ) & $=8.716$ & IBI (India) & $=4.260$ \\
\hline & JIF & $=1.500$ & SJIF (Morocco & $=\mathbf{5 . 6 6 7}$ & OAJI (USA) & $=0.350$ \\
\hline
\end{tabular}

\section{SOI: 1.1/TAS DOI: 10.15863/TAS International Scientific Journal Theoretical \& Applied Science}

\author{
p-ISSN: 2308-4944 (print) e-ISSN: 2409-0085 (online) \\ Year: 2019 Issue: $04 \quad$ Volume: 72
}

Published: $30.04 .2019 \quad$ http://T-Science.org
QR - Issue

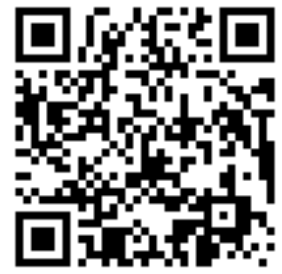

Mukhammadyunus Usubzhonovich Norinov Doctoral student,

Tashkent University of information technologies named after Muhammad al-Khorezm

Bakhtier Abduvakhidovich Abdukodirov Senior lecturer,

Fergana branch of Tashkent University of information technologies named after Muhammad al-Khorezm

\section{Azizbek Otabekovich Tillavoldiev}

Assistant,

Fergana branch of Tashkent University of information technologies named after Muhammad al-Khorezm

Nodirbek Toxirjonovich Urinov Assistant,

Andijan state University

\title{
ALGORITHM FOR ELIMINATING NOISE BY A SMOOTH-SMOOTH IMAGE MODEL
}

Abstract: The article analyzes the existing methods and technologies to eliminate possible noise or noise. An algorithm for adaptive median filtering is proposed, designed to attenuate a more intense bipolar impulse noise, as well as a method for eliminating noise by a piecewise-smooth image model. A method of image processing by adaptive low-frequency filtering has been developed, which allows to significantly improve the image quality.

Key words: impulse, intensity, signal filtering, noise, piecewise smooth model.

Language: Russian

Citation: Norinov, M. U., Abdukodirov, B. A., Tillavoldiev, A. O., \& Urinov, N. T. (2019). Algorithm for eliminating noise by a smooth-smooth image model. ISJ Theoretical \& Applied Science, 04 (72), 509-512.

Soi: http://s-o-i.org/1.1/TAS-04-72-65 Doi: crossef https://dx.doi.org/10.15863/TAS.2019.04.72.65

\section{АЛГОРИТМ УСТРАНЕНИЯ ШУМА КУСОЧНО-ГЛАДКОЙ МОДЕЛЬЮ ИЗОБРАЖЕНИЯ}

Аннотация: В статье проведен анализ существующих методов и технологий устранения возможных помех или шумов. Предложен алгоритм адаптивной медианной фильтрации, предназначенный для ослабления более интенсивной биполярной импульсной помехи, а также метод устранения шума кусочногладкой моделью изображения. Разработана методика обработки изображений путем адаптивной низкочастотной фильтрации, позволяющая сущеественно повысить качество изображения.

Ключевые слова: импульс, интенсивность, фильтрация сигнала, помехи, кусочно-гладкий модель.

\section{Введение}

Интенсивное развитие средств вычислительной техники и информационных технологий открыли широкой сферы их применение в обработке различного вида изображений.

В частности, расширение сферы применение информационных технологий в космонавтике, в медицине, в криминалистике, в геодезии, в картографии, в транспорте, в архиве, в телевидении и которые соответственно внесли ощутимый вклад в развитие экономики, техники и науки. Одновременно возникли и другие проблемы, связанные наличием помех или белого шума в изображениях. Поэтому приобрела необходимость разработки новых методов и 


\begin{tabular}{|c|c|c|c|c|c|c|}
\hline \multirow{4}{*}{ Impact Factor: } & ISRA (India) & $=3.117$ & SIS (USA) & $=0.912$ & ICV (Poland) & $=6.630$ \\
\hline & ISI (Dubai, UAE & $=0.829$ & РИНЦ (Russia & $=\mathbf{0 . 1 5 6}$ & PIF (India) & $=1.940$ \\
\hline & GIF (Australia) & $=0.564$ & ESJI (KZ) & $=8.716$ & IBI (India) & $=4.260$ \\
\hline & JIF & $=1.500$ & SJIF (Morocco & $=5.667$ & OAJI (USA) & $=0.350$ \\
\hline
\end{tabular}

технологий устранения возможных помех или шумов.

\section{Основная часть}

Алгоритм адаптивной медианной фильтрации предназначен для ослабления более интенсивной биполярной импульсной помехи, вероятность появления импульсов которой превышает $p_{n} \leq 0,2$ [1]. Кроме того, этот алгоритм обладает тем преимуществом, что он в меньшей степени искажает детали изображения, не поврежденные импульсным шумом. Особенностью адаптивного алгоритма является то, что в отличие от обычного медианного фильтра он при определенных условиях увеличивает размер окна, перекрывающего нечетное число пикселов, которым сканируется фильтруемое изображение [4]. При реализации алгоритма измеряются следующие значения интенсивностей пикселов, оказавшихся в пределах окна, которое, как и ранее, может иметь любую форму (прямоугольную, крестообразную и т. д.):

- максимальное значение интенсивности $L_{\text {макс }}$

- минимальное значение интенсивности (яркости) $L_{\text {мин }}$;

- значение интенсивности пиксела, занимающего центральное положение в окне $L_{c}$;

- медиана последовательности пикселов, оказавшихся в окне $L_{\text {мед }}$;

- максимальный допустимый размер

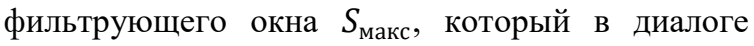
задается числом пикселов.

Алгоритм адаптивной медианной фильтрации включает в себя две ветви: I и II. Задача, которую выполняет первая ветвь, - это определение, является ли медиана $L_{\text {мед }}$ результатом воздействия импульса помехи (положительного или отрицательного) на изображение, или нет. В том случае если выполняется условие $L_{\text {мин }}<L_{\text {мед }}<L_{\text {макс }}$, то

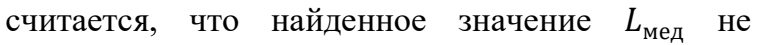
является результатом воздействия импульса помехи на изображение, и тогда совершается переход к выполнению второй ветви алгоритма. При выполнении второй ветви алгоритма проверяется, является ли значение интенсивности пиксела, занимающего центральное положение в окне, $L_{c}$, результатом воздействия импульса помехи (положительного или отрицательного) на изображение, или нет [5]. *В том случае если выполняется условие $L_{\text {мин }}<L_{\mathrm{c}}<L_{\text {макс }}$, то считается, что значение $L_{\mathrm{c}}$, не является результатом воздействия импульса помехи на изображение, и в качестве результата фильтрации принимается значение $L_{c}$, а не значение медианы. Благодаря этому минимизируются искажения, неизбежно возникающие при фильтрации изображения. В том случае, если это неравенство не выполняется, т. е. либо $L_{\mathrm{c}}=L_{\text {макс }}$ либо $L_{\mathrm{c}}=L_{\text {мин }}$, считается, что это результат воздействия импульса помехи на изображение, и в качестве результата фильтрации принимается значение $L_{\text {мед}}$, которое, как следует из результата работы первой ветви алгоритма, не является следствием воздействия импульса помехи.

Продолжая изложение работы алгоритма, рассмотрим случай, когда при выполнении первой ветви алгоритма условие $L_{\text {мин }}<L_{\text {мед }}<L_{\text {макс }}$ оказывается нарушенным, т. е. случай, когда медиана считается результатом воздействия импульса помехи на изображение. В этом случае согласно алгоритму, увеличиваются размеры фильтрующего окна и повторяются вычисления первой ветви алгоритма. Так будет продолжаться до тех пор, пока либо не будет найдена медиана, не считающаяся результатом воздействия импульса помехи, либо размеры окна не достигнут максимально разрешенного размера $S_{\text {макс }}$ В последнем случае в качестве результата фильтрации принимается значение $L_{c}$ [3].

Далее приводится схема алгоритма.

Ветвь I:

$$
\begin{aligned}
& A 1=L_{\text {мед }}-L_{\text {мин }} ; \\
& A 2=L_{\text {мед }}-L_{\text {макс }} ;
\end{aligned}
$$

если $\mathrm{A} 1>0$ и $\mathrm{A} 2<0$, то перейти к ветви II; иначе увеличить размер окна;

если размер окна меньше либо равен $S_{\text {макс }}$, повторить ветвь I; иначе принять в качестве результата $L_{\mathrm{c}}$.

Ветвь II:

$$
\begin{aligned}
& B 1=L_{c}-L_{\text {мин }} \\
& B 2=L_{c}-L_{\text {макс }}
\end{aligned}
$$

если В1 $>0$ и В $2<0$, принять в качестве результата $L_{c}$; иначе принять в качестве результата $L_{\text {мед. }}$.

Метод, устранения шума кусочно-гладкой моделью изображения предназначен для оценки и устранения шума из изображения в автоматическом режиме, он базируется на использовании кусочно-гладкой модели изображения (piecewise smooth image model) [2]. Алгоритм этого метода включает в себя следующие этапы.

1. Вначале зашумленное изображение $L(x, y)$ подвергается сегментации, при этом из множества известных методов сегментации авторы применяют так называемый К-метод, как это описано в [3]. В результате проведенной сегментации изображение оказывается разделенным на сегменты (области) $\Omega_{i}$. При этом каждый сегмент представляется средним значением цвета и некоторой пространственной протяженностью.

Пространственная протяженность задается таким образом, чтобы 


\begin{tabular}{|c|c|c|c|c|c|c|}
\hline \multirow{4}{*}{ Impact Factor: } & ISRA (India) & $=3.117$ & SIS (USA) & $=0.912$ & ICV (Poland) & $=6.630$ \\
\hline & ISI (Dubai, UAE & $=0.829$ & РИНЦ (Russia & $=\mathbf{0 . 1 5 6}$ & PIF (India) & $=1.940$ \\
\hline & GIF (Australia) & $=0.564$ & ESJI (KZ) & $=8.716$ & IBI (India) & $=4.260$ \\
\hline & JIF & $=1.500$ & SJIF (Morocco & $=5.667$ & OAJI (USA) & $=0.350$ \\
\hline
\end{tabular}

форма сегмента имела бы тенденцию к выпуклой форме, и чтобы все сегменты имели бы примерно одинаковые размеры.

2. Следующая операция, авторы ее называют по сегментной аффинной реконструкцией (persegment affine reconstruction), заключается в том, что каждый сегмент подвергается аффинному преобразованию, в результате которого для каждого сегмента находится функция $L_{A F}(x, y)$, определяющая распределение яркости в его пределах, для которой

$$
\sigma^{2}=\overline{\left[L(x, y)-L_{A F}(x, y)\right]^{2}}
$$

минимально. Эта функция называется аффинной реконструкцией сегмента. Далее принимается, что разность между зашумленным изображением и его аффинной реконструкцией

$$
\Delta L(x, y)=L(x, y)-L_{A F}(x, y),
$$

состоит из двух составляющих: составляющей текстуры $L_{T}(x, y)$, и составляющей шума $L_{ш}(x, y)$ :

$$
\Delta L(x, y)=L_{T}(x, y)+L_{\amalg}(x, y),
$$

Таким образом, исходное, зашумленное изображение рассматривается как сумма трех составляющих

$$
L(x, y)=L_{A F}(x, y)+L_{T}(x, y)+L_{\amalg}(x, y),
$$

при этом составляющими, представляющими не зашумлённое изображение $L_{c}(x, y)$, т.е. сигнальными, являются первые две.

$$
L_{c}(x, y)=L_{A F}(x, y)+L_{T}(x, y) \text {. }
$$
что:

Далее в цитируемой работе принимается,

- аффинная реконструкция сегмента не является случайным процессом;

- текстура и шум являются случайными взаимно некоррелированными процессами, ковариационные матрицы которых $-K_{T}$ и $K_{Ш}$ соответственно;

- сигнальный и шумовой компоненты взаимно независимы.

3. Использование аффинные реконструкции сегментов для реконструкции всего изображения в целом, то в нем возникнут ложные контуры и, кроме того, реальные границы станут более резкими. Во избежание этого производится оценка размытости границ в исходном, зашумленном изображении следующим образом [8]. Вычисляются ряд размытых версий $L_{A F \Omega}(x, y, r)$ аффинной реконструкции $L_{A F}(x, y)$ путем ее свертки с импульсной характеристикой

$$
h(x, y)=\frac{1}{\pi r^{2}} \exp \left(-\frac{x^{2}+y^{2}}{r^{2}}\right)
$$

где $r$ - параметр, определяющий степень размытия. Чем больше $r$, тем больше размытие. Затем каждая граница $C_{i j}$ между сегментами $\Omega_{i}$ и $\Omega_{j}$ расширяется в пять раз как в направлении области $\Omega_{i}$, так и в направлении области $\Omega_{j}$ для того, чтобы получить маску $\Gamma_{i j}$. После этого находятся значения средних квадратов разностей исходного изображения $L(x, y)$ и его размытых версий $L_{A F \Omega}(x, y, r)$ для каждого значения параметра $r$ в пределах маски, т. е.

$$
\sigma^{2}(r)=\overline{\left[L(x, y)-L_{A F \Omega}(x, y, r)\right]^{2}},
$$

За значение параметра, характеризующего степень размытия в исходном изображении, принимается то, обозначим его $r_{\text {опт, }}$ которому соответствует минимум среднего квадрата $\overline{\left[L(x, y)-L_{A F \Omega}(x, y, r)\right]^{2}}, \quad$ вычисленного в пределах маски $\Gamma_{i j}$. . После этого производится замена неразмытых границ в пределах, определяемых маской $\Gamma_{i j}$, на размытые границы, взятые из аффинной реконструкции $L_{A F \Omega}(x, y, r)$ полученной при найденном параметре размытия $r_{\text {опт }}$.

4. Далее, применяя Байесовский подход к решению задачи, находятся апостериорные оценки ковариационных матриц шума $K_{\text {аш }}$ и текстуры $K_{\text {ат }}$ соответственно [7].

5. Заключительным этапом алгоритма является реконструкция обрабатываемого изображения. Для этой цели авторы используют: исходное, зашумленное изображение, его аффинную реконструкцию, полученную при найденном параметре размытия $r_{\text {опт, }}$ а также апостериорные оценки матриц шума и текстуры.

Описанный метод действительно является эффективным в отношении подавления шума;

\section{Заключение}

Разработана методика обработки изображений путем адаптивной низкочастотной фильтрации, позволяющая существенно повысить качество изображения, отличающаяся применением низкочастотных фильтров в зависимости от типа шума на изображении.

Модифицирован метод выделения контуров изображения путем вычисления градиента, который отличается большей точностью вычисления градиента изображения. 


\begin{tabular}{|c|c|c|c|c|c|c|}
\hline \multirow{4}{*}{ Impact Factor: } & ISRA (India) & $=3.117$ & SIS (USA) & $=0.912$ & ICV (Poland) & $=6.630$ \\
\hline & ISI (Dubai, UAE & $=0.829$ & РИНЦ (Russia & $=\mathbf{0 . 1 5 6}$ & PIF (India) & $=1.940$ \\
\hline & GIF (Australia) & $=0.564$ & ESJI (KZ) & $=8.716$ & IBI (India) & $=4.260$ \\
\hline & JIF & $=1.500$ & SJIF (Morocco & $=5.667$ & OAJI (USA) & $=0.350$ \\
\hline
\end{tabular}

\section{References:}

1. Gruzman, I. S. (2000). Tsifrovaya obrabotka izobrazheniy $v$ informatsionnykh sistemakh: Uchebnoe posobie. (p.168). Novosibirsk: Izd-vo NGTU.

2. Bezrukov, B. N. (2002). Spetsifika videokontrolya izobrazheniy veshchatel'nogo televideniya. Materialy mezhdunarodnogo kongressa HAT, Moskva, pp.215-216.

3. Veligosha, D. A. (2010). Algoritm szhatiya izobrazheniy na osnove veyvlet-preobrazovaniy. Izv. Orel GTU. Inf. sistemy i tekhnol., №5, pp. 13-16.

4. Abramov, S. K., Fevralev, D. V., Roenko, A. A., Zryakhov, M. S., \& Lukin, V. V. (2011). Obrabotka informatsii $v$ tsifrovykh sistemakh svyazi. Uchebnoe posobie po laboratornomu praktikumu. (p.38, p.1). Khar'kov: KhAI.

5. Belikova, T. P., Stenina, I. I., \& Yashunskaya, N. I. (1997). Obrabotka izobrazheniy i sindromnyy analiz priznakov dlya uluchsheniya izobrazheniy. Komp'yuternaya optika, № 17, pp. 103-111.

6. Betkhin, Y. S., \& Bryantsev, A. A. (2012). Kompressiya zashumlennykh izobrazheniy $\mathrm{V}$ biblioteke veyvlet-kodekov. Vestnik RGRTU, №1. Riga, pp.146-147.

7. Shapiro, L., \& Stokman, D. (2006). Komp'yuternoe zrenie. (p.752). Moscow: BINOM. Laboratoriya znaniy.

8. Gonsales, R., \& Vuds, R. (2005). Tsifrovaya obrabotka izobrazheniy. (p.1072). Moscow: Tekhnosfera.

9. Belyaev, E. A., \& Tyurlikov, A. M. (2007). Upravlenie skorost'yu i oshibkoy kodirovaniya $\mathrm{v}$ sisteme szhatiya i peredachi videoinformatsii s ogranicheniyami na pamyat' peredayushchego i prinimayushchego ustroystv. Komp'yuternaya optika. Moskva, №2, pp.69-76.

10. Cherepanova, A. V. (2011). Otsenka kachestva szhatoy videoinformatsii. Vesti. SibGUTI, № 1, pp.61-70. 